Trinity University

Digital Commons @ Trinity

Mathematics Faculty Research

Mathematics Department

$11-2001$

\title{
Marginal and Parametric Analysis of the Central Optimal Solution
}

\author{
Allen G. Holder \\ Trinity University, aholder@trinity.edu \\ J F. Sturm \\ S Zhang
}

Follow this and additional works at: https://digitalcommons.trinity.edu/math_faculty

Part of the Mathematics Commons

\section{Repository Citation}

Holder, A.G., Sturm, J.F., \& Zhang, S. (2001). Marginal and parametric analysis of the central optimal solution. Infor, 39, 394-415. doi:10.1080/03155986.2001.11732450

This Post-Print is brought to you for free and open access by the Mathematics Department at Digital Commons @ Trinity. It has been accepted for inclusion in Mathematics Faculty Research by an authorized administrator of Digital Commons @ Trinity. For more information, please contact jcostanz@trinity.edu. 


\title{
CENTRAL OPTIMAL SOLUTION
}

\author{
A.G. Holder ${ }^{1}$, J.F. Sturm ${ }^{2}$ and S. Zhang ${ }^{3}$
}

Revision - September, 1999

\begin{abstract}
In this paper we investigate the sensitivity analysis of the parameterized central path. First, a complete marginal analysis of the central optimal solution is developed. This analysis explains the differential properties of the central optimal solution with respect to both the cost coefficients and the right-hand side components. We also show that the marginal derivatives are uniformly bounded. Second, we present three conditions for which the parameterized central path converges. Two of these results allow the difficult situation of simultaneous perturbations in the cost coefficients and right-hand side levels.
\end{abstract}

Key words: Parametric linear programming, sensitivity analysis, analytic central path.

AMS subject classification: $90 \mathrm{C} 05,90 \mathrm{C} 31$.

${ }^{1}$ Department of Mathematics, Trinity University, San Antonio, U.S.A.

2 Department of Quantitative Economics, Maastricht University, Maastricht, Netherlands. Research performed at Erasmus University Rotterdam and McMaster University, Ontario; partly supported by the Netherlands Organization for Scientific Research (NWO).

${ }^{3}$ Chinese University of Hong Kong. Research performed at Econometric Institute, Erasmus University, Rotterdam, The Netherlands. 


\section{Introduction}

Sensitivity analysis and parametric programming play an important role in linear programming. In some cases, knowing how an optimum changes relative to perturbations in the input data is more important than simply computing an optimal solution. Indeed, the data of a given problem can never be absolutely accurate in real applications. Hence, it is crucial to keep track of how optimal solutions, or the optimal value, change if the data changes. Because of this, many researchers have investigated the area of sensitivity analysis; for recent advances see Gal and Greenberg [13]. There are several software packages, many of them developed in-house by large companies, that provide aid to practitioners when trying to understand a specific solutions characteristics. As an example we mention ANALYZE [9], which has been used by the Energy Information Administration, British Telecommunications, and Amoco Oil.

We make the distinction between the following two questions:

- How does an optimal solution behave with respect to data perturbations?

- How does the optimal objective value behave with respect to data perturbations?

Both questions are completely addressed from a basic solution perspective. However, interior point algorithms for linear programming terminate with a solution, called the central optimal solution, that has different characteristics from that of a basic optimal solution, and there are circumstances under which the central optimal solution is more desirable than a basic optimal solution [10, 18, 34]. The parametric analysis of the optimal value is developed using the central optimal solution in [27]. Monteiro and Mehrotra [22] and Roos and Terlaky [28] independently developed an algorithm that uses the central optimal solution to completely describe the objective function along any single direction of change in either the cost coefficients or the right-hand side vector. This algorithm also produces the unique optimal partition as the data is changed. Greenberg [11] shows that the question of how the objective function responds to simultaneous changes in cost coefficients and right-hand side levels may be answered using the central optimal solution. All of the above analysis deals with information that is attainable from asking the second question.

We address the first question by discussing how the central optimal solution behaves when perturbations occur in either the objective coefficients or the right-hand side levels. Specifically, we present an analysis of both the marginal properties of the central optimal solution and the convergence properties of the parameterized central path. The organization of the paper is as follows. Section 2 presents the notation and terminology used throughout. Sections 3 and 4 present the marginal and parametric analysis, respectively. Section 5 concludes with a summary of the results. 


\section{Notation and Terminology}

For the scalars $\theta_{b}$ and $\theta_{c}$, consider the following standard form linear program:

$$
\begin{array}{lll}
(L P)_{\left(\theta_{b}, \theta_{c}\right)} & \text { minimize } & \left(c+\theta_{c} \delta c\right)^{T} x \\
& \text { subject to } & A x=b+\theta_{b} \delta b \\
& & x \geq 0
\end{array}
$$

and its dual problem

$$
\begin{array}{lll}
(L D)_{\left(\theta_{b}, \theta_{c}\right)} & \text { maximize } & \left(b+\theta_{b} \delta b\right)^{T} y \\
& \text { subject to } & A^{T} y+s=c+\theta_{c} \delta c \\
& s \geq 0
\end{array}
$$

where $A \in \mathbb{R}^{m \times n}, m \leq n, b+\theta_{b} \delta b \in \mathbb{R}^{m}$, and $c+\theta_{c} \delta c \in \mathbb{R}^{n}$. The vectors $\delta b$ and $\delta c$ are called the right-hand side direction of change and the cost coefficient direction of change, respectively. Similarly, the non-negative scalars $\theta_{b}$ and $\theta_{c}$ are referred to as the right-hand side parameter and the cost coefficient parameter. The primal feasible region is denoted by $\mathcal{P}_{\theta_{b}}$, and the dual feasible region is denoted by $\mathcal{D}_{\theta_{c}}$. The strict interiors of these sets are $\mathcal{P}_{\theta_{b}}^{o}$ and $\mathcal{D}_{\theta_{c}}^{o}$, defined by $\left\{x \in \mathcal{P}_{\theta_{b}}: x>0\right\}$ and $\left\{(y, s) \in \mathcal{D}_{\theta_{c}}: s>0\right\}$. Furthermore, the primal and dual optimality sets are $\mathcal{P}_{\left(\theta_{b}, \theta_{c}\right)}^{*}$ and $\mathcal{D}_{\left(\theta_{b}, \theta_{c}\right)}^{*}$

We say the input data, $(b, c)$, is admissible if both $(L P)_{(0,0)}$ and $(L D)_{(0,0)}$ satisfy Slater's condition -i.e. $\mathcal{P}_{(0,0)}^{o} \neq \emptyset$ and $\mathcal{D}_{(0,0)}^{o} \neq \emptyset$. Moreover, $(\delta b, \delta c)$ is an admissible direction of change if there exists $\theta^{*}>0$ such that $(b, c)+\theta(\delta b, \delta c)$ is admissible for all $\theta \in\left[0, \theta^{*}\right)$. For convenience, we define

$$
\begin{aligned}
\mathcal{H} & \equiv\{(\delta b, \delta c):(\delta b, \delta c) \text { is an admissible direction of change }\} \\
\mathcal{H}_{b} & \equiv\{\delta b:(\delta b, 0) \text { is an admissible direction of change }\}, \text { and } \\
\mathcal{H}_{c} & \equiv\{\delta c:(0, \delta c) \text { is an admissible direction of change }\} .
\end{aligned}
$$

Throughout, we assume that both $(b, c)$ and $(\delta b, \delta c)$ are admissible. It is easy to show that Slater's condition implies that $\mathcal{H}_{c}=\mathbb{R}^{n}$. However, because we do not assume that $A$ has full row rank, Slater's condition does not imply that $\mathcal{H}_{b}=\mathbb{R}^{m}$. As an illustration, consider a standard transportation problem with equality constraints. A right-hand side direction of change that implies an increase in demand without a corresponding increase in supply is not admissible. What is true, and rather easy to show, is that $\mathcal{H}_{b}$ is the column space of $A$. Denote the rank of $A$ by $\bar{m}$, and let $\bar{A}$ be an $\bar{m} \times n$ matrix consisting of $\bar{m}$ independent rows of $A$. Denote the corresponding subvectors of $y, b$ and $\delta$ by $\bar{y}, \bar{b}$, and $\bar{\delta}$, respectively. Furthermore, denote the remaining entries of $y$ by $\hat{y}$,

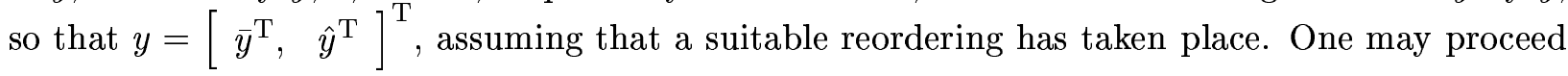
with the $(L P)$ problem

$$
\min \left\{\left(c+\theta_{c} \delta c\right)^{T} x: \bar{A} x=\bar{b}+\theta_{b} \bar{\delta} b, x \geq 0\right\}
$$


and its dual

$$
\left.\max \left\{\left(\bar{b}+\theta_{b} \bar{\delta}\right)\right)^{T} \bar{y}: \bar{A}^{T} \bar{y}+s=c+\theta_{c} \delta c, s \geq 0\right\} .
$$

However, we prefer to establish our results with original model $(L P)_{\left(\theta_{b}, \theta_{c}\right)}$, where $A$ may not have full row rank.

Each of $\mathcal{H}, \mathcal{H}_{b}$, and $\mathcal{H}_{c}$ have a subset of interest that relies on the concept of the optimal partition. Define

$$
\begin{aligned}
& B\left(\theta_{b}, \theta_{c}\right)=\left\{i: \text { there exists } x \in \mathcal{P}_{\left(\theta_{b}, \theta_{c}\right)}^{*} \text { such that } x_{i}>0\right\} \text { and } \\
& N\left(\theta_{b}, \theta_{c}\right)=\{1,2,3, \ldots, n\} \backslash B\left(\theta_{b}, \theta_{c}\right) .
\end{aligned}
$$

The sets $B\left(\theta_{b}, \theta_{c}\right)$ and $N\left(\theta_{b}, \theta_{c}\right)$ form the optimal partition and completely define the optimal sets as follows [28],

$$
\mathcal{P}_{\left(\theta_{b}, \theta_{c}\right)}^{*}=\left\{x \in \mathcal{P}_{\theta_{b}}: x_{N\left(\theta_{b}, \theta_{c}\right)}=0\right\} \quad \text { and } \quad \mathcal{D}_{\left(\theta_{b}, \theta_{c}\right)}^{*}=\left\{(y, s) \in \mathcal{D}_{\theta_{c}}: s_{B\left(\theta_{b}, \theta_{c}\right)}=0\right\} \text {, }
$$

where a set subscript is used to denote the sub-vector corresponding to the indices contained in the set. Let $\mathcal{H}^{1}, \mathcal{H}_{b}^{1}$, and $\mathcal{H}_{c}^{1}$ be respective subsets of $\mathcal{H}, \mathcal{H}_{b}$, and $\mathcal{H}_{c}$, where a direction of change being in one of these subsets implies that the optimal partition remains intact for sufficiently small amounts of perturbation. In other words, $\mathcal{H}^{1}, \mathcal{H}_{b}^{1}$, and $\mathcal{H}_{c}^{1}$ are the admissible directions of change for which the optimal sets are invariant with respect to small amounts of data change. Investigations into the dimensions of these subsets are found in [14], and a geometric description is found in Figure 1. Denoting the column and row spaces of a matrix, $M$, by $\operatorname{coll}(M)$ and $\operatorname{row}(M)$, we have the following equalities $[11,16]$ :

$$
\mathcal{H}_{b}^{1}=\operatorname{coll}\left(A_{B}\right), \quad \mathcal{H}_{c}^{1}=\operatorname{row}\left(\left[\begin{array}{cc}
A_{B} & A_{N} \\
0 & I
\end{array}\right]\right), \quad \mathcal{H}^{1}=\mathcal{H}_{b}^{1} \times \mathcal{H}_{c}^{1} .
$$

A pertinent implication of $(L P)_{(0,0)}$ and $(L D)_{(0,0)}$ satisfying Slater's condition is that both $(L P)_{\left(\theta_{b}, \theta_{c}\right)}$ and $(L D)_{\left(\theta_{b}, \theta_{c}\right)}$ satisfy Slater's condition for $\left(\theta_{b}, \theta_{c}\right)$ in a neighborhood of zero [26]. Let $\hat{\theta}_{b}$ and $\hat{\theta}_{c}$ be such that $(L P)_{\left(\theta_{b}, \theta_{c}\right)}$ and $(L D)_{\left(\theta_{b}, \theta_{c}\right)}$ satisfy Slater's condition for $\left(\theta_{b}, \theta_{c}\right)$ in $\left[0, \hat{\theta_{b}}\right) \times\left[0, \hat{\theta_{c}}\right)$. It is well known that under Slater's condition, the analytic central path exists [28]. This means that for any fixed $\left(\theta_{b}, \theta_{c}\right) \in\left[0, \hat{\theta}_{b}\right) \times\left[0, \hat{\theta}_{c}\right)$, there exist a unique $x(\mu) \in \mathcal{P}_{\theta_{b}}^{o}$ and a unique $(y(\mu), s(\mu)) \in$ $\left\{(y, s) \in \mathcal{P}_{\theta_{c}}^{o}: \hat{y}=0\right\}$ such that

$$
x_{i}(\mu) s_{i}(\mu)=\mu, \quad i=1,2, \ldots, n \text {. }
$$

The above notation indicates a reliance only on $\mu$, which is too restrictive for our purposes. We extend this notation to include a reliance on both $\theta_{b}$ and $\theta_{c}$. Formally, the parameterized central path is defined by

$$
x\left(\theta_{b}, \theta_{c}, \mu\right) \text { and }\left(y\left(\theta_{b}, \theta_{c}, \mu\right), s\left(\theta_{b}, \theta_{c}, \mu\right)\right)
$$



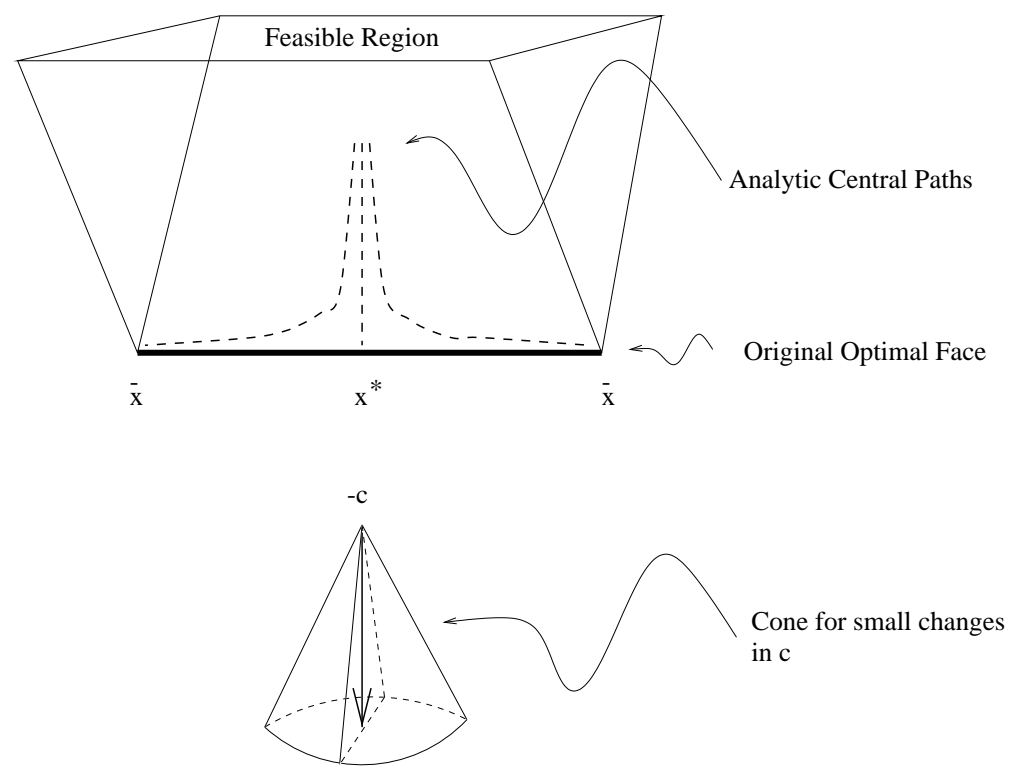

Figure 1: The cut through the cone of cost coefficient changes indicates the perturbations that leave the original optimal partition intact. Changes in $c$ not in this cut cause the central optimal solution to change from $x^{*}$ to one of the $\bar{x}$ 's.

where these vectors satisfy

$$
\left.\begin{array}{rl}
x\left(\theta_{b}, \theta_{c}, \mu\right) & \in \mathcal{P}_{\theta_{b}}^{o}, \\
\left(y\left(\theta_{b}, \theta_{c}, \mu\right), s\left(\theta_{b}, \theta_{c}, \mu\right)\right) & \in \mathcal{D}_{\theta_{c}}^{o}, \\
\hat{y}\left(\theta_{b}, \theta_{c}, \mu\right)=0, \text { and } & \\
x_{i}\left(\theta_{b}, \theta_{c}, \mu\right) s_{i}\left(\theta_{b}, \theta_{c}, \mu\right) & =\mu, i=1,2, \ldots, n .
\end{array}\right\}
$$

Since the work of Sonnevend [30], the elements of the parameterized central path have been known to be analytic functions not only of $\mu, \mu>0$, but also of $\theta_{b}$ and $\theta_{c}$. Hence, $x\left(\theta_{b}, \theta_{c}, \mu\right)$ and $s\left(\theta_{b}, \theta_{c}, \mu\right)$ are analytic functions for $\mu>0$.

It is useful to notice that the conditions in (2.2) are the necessary and sufficient Lagrange conditions showing that $x\left(\theta_{b}, \theta_{c}, \mu\right)$ is the unique minimizer of

$$
\min \left\{\left(c+\theta_{c} \delta c\right)^{T} x-\mu \sum_{i=1}^{n} \ln \left(x_{i}\right): x \in \mathcal{P}_{\theta_{b}}^{o}\right\} \text {. }
$$

Here, $\sum_{i=1}^{n} \ln \left(x_{i}\right)$ is the logarithmic barrier function, and $\mu$ is called the barrier parameter. One of the well established properties of the analytic central path is that it converges to the central optimal solution as the barrier parameter decreases to zero [19]. We define these limits by,

$$
x^{*}\left(\theta_{b}, \theta_{c}\right) \equiv \lim _{\mu \downarrow 0} x\left(\theta_{b}, \theta_{c}, \mu\right) \text { and }
$$




$$
\left(y^{*}\left(\theta_{b}, \theta_{c}\right), s^{*}\left(\theta_{b}, \theta_{c}\right)\right) \equiv \lim _{\mu \downarrow 0}\left(y\left(\theta_{b}, \theta_{c}, \mu\right), s\left(\theta_{b}, \theta_{c}, \mu\right)\right) .
$$

Furthermore, these limits are the analytic centers, respectively, of $\mathcal{P}_{\left(\theta_{b}, \theta_{c}\right)}^{*}$ and $\mathcal{D}_{\left(\theta_{b}, \theta_{c}\right)}^{*}$. That is, $x^{*}\left(\theta_{b}, \theta_{c}\right)$ is the unique solution to

$$
\max \left\{\sum_{i \in B\left(\theta_{b}, \theta_{c}\right)} \ln \left(x_{i}\right): A x=b+\theta_{b} \not b, x_{B\left(\theta_{b}, \theta_{c}\right)}>0, x_{N\left(\theta_{b}, \theta_{c}\right)}=0\right\}
$$

and $\left(y^{*}\left(\theta_{b}, \theta_{c}\right), s^{*}\left(\theta_{b}, \theta_{c}\right)\right)$ solves

$$
\max \left\{\sum_{i \in N\left(\theta_{b}, \theta_{c}\right)} \ln \left(s_{i}\right): A^{T} y+s=c+\theta_{c} \delta c, s_{N\left(\theta_{b}, \theta_{c}\right)}>0, s_{B\left(\theta_{b}, \theta_{c}\right)}=0\right\} .
$$

The standard operator notation, $D$, is used for differentiation. If $f: \mathbb{R}^{n} \rightarrow \mathbb{R}^{m}$ is $k$ times differentiable in a neighborhood of $u \in \mathbb{R}^{n}$, then $D_{u_{i}}^{k} f(u)$ is the vector whose $j^{\text {th }}$ component is $\frac{\partial^{k} f_{j}}{\partial u_{i}^{k}}$, where $f_{j}$ is the $j^{\text {th }}$ component function.

In this paper, we study the existence and value of $D_{\theta_{b}}^{k} x^{*}\left(\theta_{b}, 0\right)$ and $D_{\theta_{c}}^{k} x^{*}\left(0, \theta_{c}\right)$. We extend the notation as follows,

$$
\begin{gathered}
D_{\theta_{b}}^{k} x^{*}\left(0^{+}, 0\right)=\lim _{\theta_{b} \downarrow 0} D_{\theta_{b}}^{k} x^{*}\left(\theta_{b}, 0\right), \text { and } \\
D_{\theta_{b}^{+}}^{k} x^{*}(0,0)=\lim _{\theta_{b} \downarrow 0} \frac{D_{\theta_{b}}^{k-1} x^{*}\left(\theta_{b}, 0\right)-D_{\theta_{b}}^{k-1} x^{*}(0,0)}{\theta_{b}},
\end{gathered}
$$

where $D_{\theta_{b}}^{k-1} x^{*}(0,0) \equiv D_{\theta_{b}}^{k-1} x^{*}\left(0^{+}, 0\right)$. Analogous definitions hold for $D_{\theta_{c}}^{k} x^{*}\left(0,0^{+}\right)$and $D_{\theta_{c}^{+}}^{k} x^{*}(0,0)$. For convenience, we let $D^{0}$ be the identity operator. The derivatives of a real valued, single variable function, $g$, are denoted by $g^{\prime}, g^{\prime \prime}, \ldots, g^{(k)}$. The right sided derivatives and limiting derivatives are written, $g_{+}^{\prime}, g_{+}^{\prime \prime}, \ldots, g_{+}^{(k)}$ and $g^{\prime}\left(x_{0}^{+}\right), g^{\prime \prime}\left(x_{0}^{+}\right), \ldots, g^{(k)}\left(x_{0}^{+}\right)$, respectively. The set of infinitely differentiable functions is denoted by $\mathcal{C}^{\infty}$. We say that an analytic property holds locally at $x$ if there exists an open neighborhood of $x$ for which the property holds.

Given a vector $x \in \mathbb{R}^{n}$, we implicitly let the upper case symbol $X$ denote the $n \times n$ diagonal matrix with the elements of $x$ on its diagonal. For terminology that is common in the linear programming literature, we refer to the Math Programming Glossary [12].

\section{Marginal Analysis of the Central Optimal Solution}

In this section we investigate the marginal, or differential, properties of the central optimal solution. The marginal analysis of $x^{*}\left(0, \theta_{c}\right)$ along $\delta c$ is quite simple because, as shown in Theorem 3.1, $x^{*}\left(0, \theta_{c}\right)$ is a step function along $\delta$. However, the differential properties of $x^{*}\left(\theta_{b}, 0\right)$ along $\delta b$ are 
more intricate, and our analysis is two fold in it objectives. First, we show that the central optimal solution is a continuous, piecewise analytic function along $\delta b$. Second, we provide uniform bounds for the derivatives of the central optimal solution. We begin with the following monotonicity relationship for the optimal partition, which is found in both $[2,3]$.

Lemma 3.1 (Adler and Monteiro [2]) For sufficiently small $\left(\theta_{b}, \theta_{c}\right)>0$, we have

$$
N\left(\theta_{b}, 0\right) \subseteq N(0,0) \subseteq N\left(0, \theta_{c}\right) \text { and } B\left(0, \theta_{c}\right) \subseteq B(0,0) \subseteq B\left(\theta_{b}, 0\right)
$$

In addition to Lemma 3.1, Greenberg [11] has shown that if $\theta_{c}=0$, there exists $\theta_{b}^{*}>0$ such that the optimal partition is invariant for $\theta_{b} \in\left(0, \theta_{b}^{*}\right)$. Similarly, if $\theta_{b}=0$, there exists $\theta_{c}^{*}>0$ such that the optimal partition does not change for any $\theta_{c} \in\left(0, \theta_{c}^{*}\right)$.

The first result of this section completely describes the behavior of the central optimal solution with respect to cost coefficient parameterization. Specifically, the following result shows that the central optimal solution is a step function along $\delta c$. Although our development follows from a primal perspective, we include for future reference the corresponding dual statement in this result. The immediate corollary states the subsequent marginal properties.

Theorem 3.1 The central optimal solution, $x^{*}\left(0, \theta_{c}\right)$, is a step function in $\theta_{c}$. Similarly, $s^{*}\left(\theta_{b}, 0\right)$ is a step function in $\theta_{b}$.

Proof. If $\delta c \in \mathcal{H}_{c}^{1}$, there exists $\theta_{c}^{*}>0$ such that the optimal partition, $\left(B\left(0, \theta_{c}\right) \mid N\left(0, \theta_{c}\right)\right)$, is invariant for any $\theta_{c} \in\left[0, \theta_{c}^{*}\right)$. Since $x^{*}\left(0, \theta_{c}\right)$ is the analytic center of $\left\{x: A x=b, x_{B(0,0)} \geq\right.$ $\left.0, x_{N(0,0)}=0\right\}$, so that $x^{*}\left(0, \theta_{c}\right)=x^{*}(0,0)$ for any $\theta_{c} \in\left[0, \theta_{c}^{*}\right)$. If $\delta \notin \notin \mathcal{H}_{c}^{1}$, there exists $\theta_{c}^{*}>$ 0 such that the optimal partition, $\left(B\left(0, \theta_{c}\right) \mid N\left(0, \theta_{c}\right)\right)$, is invariant with respect to $\theta_{c} \in\left(0, \theta_{c}^{*}\right)$. From Lemma 3.1, $N(0,0) \subset N\left(0, \theta_{c}\right)$ and $B\left(0, \theta_{c}\right) \subset B(0,0)$. Let $\mathcal{I}=B(0,0) \backslash B\left(0, \theta_{c}\right)$. From (2.3), we have that $x_{\mathcal{I}}^{*}(0,0)>0$. However, for all $\theta_{c} \in\left(0, \theta_{c}^{*}\right), x_{\mathcal{I}}^{*}\left(0, \theta_{c}\right)=0$, which implies a discontinuity at $\theta_{c}=0$. Furthermore, for any of the finite number of partitions, $(D \mid F)$, of $\{1,2, \ldots, n\},\left\{\theta_{c}:\left(B\left(0, \theta_{c}\right) \mid N\left(0, \theta_{c}\right)\right)=(D \mid F)\right\}$ is convex [11], which implies there is only a finite number of discontinuities. The dual statement follows from an analogous argument. $\quad$ Q.E.D.

Corollary 3.1 If $\delta \in \in \mathcal{H}_{c}^{1}, D_{\theta_{c}}^{k} x^{*}(0,0)=0$. Otherwise, $x^{*}\left(0, \theta_{c}\right)$ is discontinuous at $\theta_{c}=0$.

Proof. From $(2.1), \delta c \in \mathcal{H}_{c}^{1}$ implies $-\delta c \in \mathcal{H}_{c}^{1}$. So $\left(B\left(0, \theta_{c}\right) \mid N\left(0, \theta_{c}\right)\right)=(B(0,0) \mid N(0,0))$ for all $\theta_{c}$ in some neighborhood of 0 . Furthermore, as indicated in the prior proof, $x^{*}\left(0, \theta_{c}\right)$ is invariant over this neighborhood. Hence, $D_{\theta_{c}}^{k} x^{*}(0,0)=0$, for all $k$. The case when $\delta c \in \mathcal{H}_{c} \backslash \mathcal{H}_{c}^{1}$ is addressed in the proof of Theorem 3.1.

Q.E.D. 
Similar to Corollary 3.1, the marginal analysis with respect to the right-hand side parameter depends on whether or not $\delta b \in \mathcal{H}_{b}^{1}$. However, unlike the result stated in Corollary 3.1, the analytic center solution is continuous along any admissible 86 . In fact, the analytic center solution is a continuous, piecewise analytic function with respect to linear changes in the right-hand side. The next result, which is a direct consequence of the implicit function theorem [7], establishes that the analytic center solution is an analytic function of $\theta_{b}$, provided that the optimal partition remains intact.

Theorem 3.2 If $\delta \in \mathcal{H}_{b}^{1}, x^{*}\left(\theta_{b}, 0\right)$ is locally analytic in $\theta_{b}$.

Proof. From (2.1), $\delta b \in \mathcal{H}_{b}^{1}$ implies $-\delta b \in \mathcal{H}_{b}^{1}$. Hence, $(B(0,0) \mid N(0,0))=\left(B\left(\theta_{b}, 0\right) \mid N\left(\theta_{b}, 0\right)\right)$ for $\theta_{b}$ in some sufficiently small neighborhood of 0 , and $x_{N\left(\theta_{b}, 0\right)}^{*}\left(\theta_{b}, 0\right)=x_{N(0,0)}^{*}(0,0)=0$ over this same neighborhood. Since $\delta b \in \operatorname{coll}\left(A_{B}\right)$, we have that $\left\{x: A_{B} x=b+\theta_{b} \delta b\right\}=\left\{x: \bar{A}_{B} x=\bar{b}+\theta_{b} \overline{\delta b}\right\}$. The analytic center solution of the unperturbed problem is the unique solution to the following system at $\theta_{b}=0$,

$$
\begin{aligned}
\bar{A}_{B} x_{B} & =\bar{b}+\theta_{b} \overline{\delta b} \\
\bar{A}_{B}^{T} \bar{y}+s_{B} & =0 \\
X_{B} s_{B} & =e \\
x_{B} & >0 \\
s_{B} & >0
\end{aligned}
$$

(Recall that $X_{B}$ is the diagonal matrix with the elements of $x_{B}$ on its diagonal.) Denote the solution to (3.5)-(3.9), with $\theta=0$, by $\left(x_{B}^{*}, \tilde{s}_{B}, \tilde{y}\right)$. Define $\phi: \mathbb{R}^{2|B|+\bar{m}+1} \rightarrow \mathbb{R}^{2|B|+\bar{m}}$ as

$$
\phi\left(x_{B}, \bar{y}, s_{B}, \theta_{b}\right)=\left[\begin{array}{c}
\bar{A}_{B} x_{B}-\theta_{b} \bar{\delta} \bar{b}-\bar{b} \\
\bar{A}_{B}^{T} \bar{y}+s_{B} \\
X_{B} s_{B}-e
\end{array}\right] .
$$

Then, $\phi$ is analytic in an open neighborhood of $\left(x_{B}^{*}, \tilde{y}, \tilde{s}, 0\right)$ and $\phi\left(x_{B}^{*}, \tilde{y}, \tilde{s}, 0\right)=0$. Since the Jacobian of $\phi\left(\left(x_{B}^{*}, \tilde{y}, \tilde{s}, 0\right)\right)$ with respect to $\left(x_{B}, \bar{y}, s_{B}\right)$ is non-singular, the implicit function theorem implies that $x_{B}$ is an analytic function of $\theta_{b}$ in some sufficiently small neighborhood of zero. $\quad$ Q.E.D.

Notice that if $\delta b$ had not been in $\operatorname{coll}\left(A_{B}\right)$, the implicit function theorem is not applicable since the needed row reduction to form $(\bar{A}, \bar{b}, \bar{\delta})$ is not possible. Theorem 3.2 implies that if we are concerned with a right-hand side direction of change that does not immediately alter the partition, not only is $x^{*}\left(\theta_{b}, 0\right)$ of class $\mathcal{C}^{\infty}$, but $x^{*}\left(\theta_{b}, 0\right)$ has a power series expansion. Differentiating (3.5), (3.6), and 
(3.7) with respect to $\theta_{b}$ and denoting $x^{*}\left(\theta_{b}, 0\right)$ as $x^{*}$, we have

$$
\begin{aligned}
\bar{A}_{B}\left(D_{\theta_{b}}^{1} x_{B}^{*}\right) & =\overline{\delta b} \\
\bar{A}_{B}^{T}\left(D_{\theta_{b}}^{1} \tilde{y}\right)+D_{\theta_{b}}^{1} \tilde{s}_{B} & =0 \\
\tilde{S}_{B}\left(D_{\theta_{b}}^{1} x_{B}^{*}\right)+X_{B}^{*}\left(D_{\theta_{b}}^{1} \tilde{s}_{B}\right) & =0 .
\end{aligned}
$$

Noticing that this is a non-singular system of linear equations in $D_{\theta_{b}}^{1} x_{B}^{*}, D_{\theta_{b}}^{1} \tilde{s}_{B}$, and $D_{\theta_{b}}^{1} \tilde{y}_{B}$, we have the following,

$$
\begin{aligned}
D_{\theta_{b}}^{1} x_{B}^{*} & =\left(X_{B}^{*}\right)^{2} \bar{A}_{B}^{T}\left(\bar{A}_{B}\left(X_{B}^{*}\right)^{2} \bar{A}_{B}^{T}\right)^{-1} \overline{\delta b}, \\
D_{\theta_{b}}^{1} \tilde{s}_{B} & =-\bar{A}_{B}^{T}\left(\bar{A}_{B}\left(X_{B}^{*}\right)^{2} \bar{A}_{B}^{T}\right)^{-1} \bar{\delta} \bar{b}, \text { and } \\
D_{\theta_{b}}^{1} \tilde{y} & =\left(\bar{A}_{B}\left(X_{B}^{*}\right)^{2} \bar{A}_{B}^{T}\right)^{-1} \bar{\delta} b .
\end{aligned}
$$

Using (3.10), we are able to recursively establish the higher order derivatives. For $k \geq 2$, define

$$
\Omega^{k} \equiv-\sum_{i=1}^{k-1}\left(\begin{array}{c}
k \\
i
\end{array}\right)\left(D_{\theta_{b}}^{i} \tilde{S}_{B}\right)\left(D_{\theta_{b}}^{k-i} x_{B}^{*}\right)
$$

Then, for $k \geq 2$ we have

$$
\begin{aligned}
\bar{A}_{B}\left(D_{\theta_{b}}^{k} x_{B}^{*}\right) & =0 \\
\bar{A}_{B}^{T}\left(D_{\theta_{b}}^{k} \tilde{y}\right)+D_{\theta_{b}}^{k} \tilde{s}_{B} & =0 \\
\tilde{S}_{B}\left(D_{\theta_{b}}^{k} x_{B}^{*}\right)+X_{B}^{*}\left(D_{\theta_{b}}^{k} \tilde{s}_{B}\right) & =\Omega^{k}
\end{aligned}
$$

which implies$$
\begin{aligned}
D_{\theta_{b}}^{k} x_{B}^{*} & =\left(X_{B}^{*}\right)^{2} \Omega^{k}-X_{B}^{*} \bar{A}_{B}^{T}\left(\bar{A}_{B}\left(X_{B}^{*}\right)^{2} \bar{A}_{B}^{T}\right)^{-1} \bar{A}_{B} X_{B}^{*} \Omega^{k} \\
D_{\theta_{b}}^{k} \tilde{s}_{B} & =\bar{A}_{B}^{T}\left(\bar{A}_{B}\left(X_{B}^{*}\right)^{2} \bar{A}_{B}^{T}\right)^{-1} \bar{A}_{B} X_{B}^{*} \Omega^{k} \\
D_{\theta_{b}}^{k} \tilde{y} & =-\left(\bar{A}_{B}\left(X_{B}^{*}\right)^{2} \bar{A}_{B}^{T}\right)^{-1} \bar{A}_{B} X_{B}^{*} \Omega^{k}
\end{aligned}
$$

Hence,

$$
x^{*}\left(\theta_{b}, 0\right)=\sum_{k=0}^{\infty} \frac{\left(D_{\theta_{b}}^{k} x^{*}(0,0)\right)}{k !}\left(\theta_{b}\right)^{k}
$$

for $\theta_{b}$ sufficiently close to zero. Notice that this power series expansion is easy to calculate to a given order, since it requires only the single matrix factorization of $\left(\bar{A}_{B}\left(X_{B}^{*}\right)^{2} \bar{A}_{B}^{T}\right)$.

We now consider the case when $\delta b \in \mathcal{H}_{b} \backslash \mathcal{H}_{b}^{1}$. In this situation, the only analytic property for $x^{*}\left(\theta_{b}, 0\right)$ that holds locally at $\theta_{b}=0$ is continuity. The following example demonstrates that even if $-\varnothing$ is admissible, the one-sided derivatives are not guaranteed to be equivalent.

Example: Consider the linear program $\min \left\{-x_{2}: 0 \leq x_{1} \leq 1,0 \leq x_{2} \leq 1, x_{1}+x_{2} \leq 1+\theta_{b}\right\}$, which is illustrated in Figure 2. After adding a slack vector,

$$
A=\left[\begin{array}{lllll}
1 & 0 & 1 & 0 & 0 \\
0 & 1 & 0 & 1 & 0 \\
1 & 1 & 0 & 0 & 1
\end{array}\right], b=\left(\begin{array}{l}
1 \\
1 \\
1
\end{array}\right), \delta=\left(\begin{array}{l}
0 \\
0 \\
1
\end{array}\right), \text { and } c=\left(\begin{array}{c}
0 \\
-1 \\
0 \\
0 \\
0
\end{array}\right)
$$




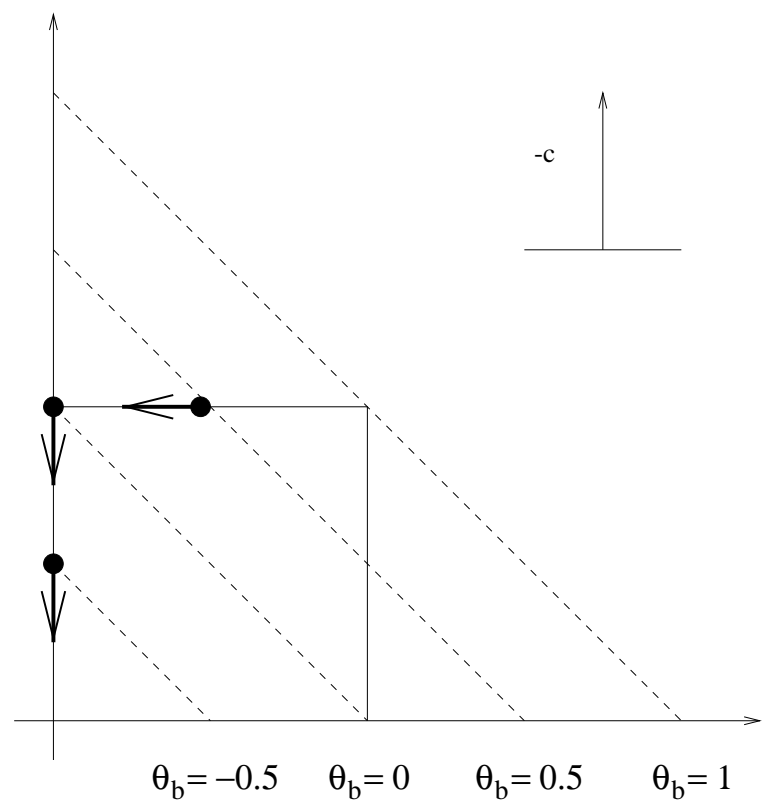

Figure 2: The movement of the central optimal solution, as $\theta_{b}$ decreases from 1 to $-1 / 2$, is indicated by the bold arrows.

The central optimal solution is

$$
\left(x_{1}^{*}\left(\theta_{b}, 0\right), x_{2}^{*}\left(\theta_{b}, 0\right)\right)= \begin{cases}\left(1+\theta_{b}-\frac{1}{3} \sqrt{\theta_{b}^{2}+3 \theta_{b}+9}, 1\right) & \text { if } \theta_{b}>0 \\ \left(0,1+\theta_{b}\right) & \text { if }-1 \leq \theta_{b} \leq 0\end{cases}
$$

and the optimal partitions for $\theta_{b}>0, \theta_{b}=0$, and $-1<\theta_{b}<0$ are respectively,

$$
(\{1,2,3,5\},\{4\}),(\{2,3\},\{1,4,5\}) \text {, and }(\{2,3,4\},\{1,5\}) \text {. }
$$

If $\theta_{b}=0, \infty b \in \mathcal{H}_{b} \backslash \mathcal{H}_{b}^{1}$ because $\$ \notin \notin \operatorname{coll}\left(A_{B}\right)$. In this situation, we have that the limiting right-sided derivative is

$$
\lim _{\theta_{b} \rightarrow 0^{+}} D_{\theta_{b}}^{1} x^{*}\left(\theta_{b}, 0\right)=\lim _{\theta_{b} \rightarrow 0^{+}}\left(\begin{array}{c}
1-\frac{2 \theta_{b}+3}{6 \sqrt{\theta_{b}^{2}+3 \theta_{b}+9}} \\
0
\end{array}\right)=\left(\begin{array}{c}
\frac{5}{6} \\
0
\end{array}\right),
$$

and the limiting left-sided derivative is

$$
\lim _{\theta_{b} \rightarrow 0^{-}} D_{\theta_{b}}^{1} x^{*}\left(\theta_{b}, 0\right)=\lim _{\theta_{b} \rightarrow 0^{-}}\left(\begin{array}{l}
0 \\
1
\end{array}\right)=\left(\begin{array}{l}
0 \\
1
\end{array}\right) .
$$

Although continuity is the strongest local property allowed when $\delta b \in \mathcal{H}_{b} \backslash \mathcal{H}_{b}^{1}$, we do establish that $x^{*}\left(\theta_{b}, 0\right)$ is one-sided, infinitely, continuously differentiable. A key ingredient in our argument is the 
well known existence result of the limiting derivatives of $x\left(\theta_{b}, \theta_{c}, \mu\right)$ with respect to $\mu[1,15,33]$. The formal statement is cited below.

Lemma 3.2 (Güler [15]) For $k=1,2,3, \ldots$, both $D_{\mu}^{k} x\left(0,0,0^{+}\right)$and $D_{\mu}^{k} s\left(0,0,0^{+}\right)$exist. Moreover,

$$
\begin{aligned}
D_{\mu}^{k} x\left(0,0,0^{+}\right) & =D_{\mu^{+}}^{k} x(0,0,0), \\
D_{\mu}^{k} s\left(0,0,0^{+}\right) & =D_{\mu^{+}}^{k} s(0,0,0), \\
D_{\mu}^{1} x_{N(0,0)}\left(0,0,0^{+}\right) & =\left(S_{N(0,0)}^{*}(0,0)\right)^{-1} e_{N(0,0)}>0, \text { and } \\
D_{\mu}^{1} s_{B(0,0)}\left(0,0,0^{+}\right) & =\left(X_{B(0,0)}^{*}(0,0)\right)^{-1} e_{B(0,0)}>0 .
\end{aligned}
$$

The above differential properties are used in conjunction with the formula for the $k$ th derivative of a composite function to establish the one-sided marginal analysis of the central optimal solution. Let $h(x)=f(g(x))$, where both $f$ and $g$ are in $\mathcal{C}^{\infty}$ on some suitable neighborhoods. Then $h^{(k)}(x)$ is

$$
\sum_{m=1}^{k} \sum \frac{k !}{j_{1} ! j_{2} ! \ldots j_{k} !} \cdot\left[\left(\frac{g^{\prime}(x)}{1 !}\right)^{j_{1}}\left(\frac{g^{\prime \prime}(x)}{2 !}\right)^{j_{2}} \ldots\left(\frac{g^{(k)}(x)}{k !}\right)^{j_{k}}\right] \cdot \frac{d^{m} f}{d y^{m}}(g(x))
$$

where the second sum is taken over all non-negative integer solutions of $\sum_{i=1}^{k} i j_{i}=k$ and $\sum_{i=1}^{k} j_{i}=m[8]$. The following lemma provides some needed differential properties.

Lemma 3.3 Let $f$ map $\left[0, \mu^{*}\right)$ onto $\left[0, \theta^{*}\right)$. Furthermore, let $f$ be in $\mathcal{C}^{\infty}$ on $\left(0, \mu^{*}\right)$, let $f^{(k)}\left(0^{+}\right)$ exist for all $k \geq 1$, and let $f^{\prime}\left(0^{+}\right)>0$. Then, there exists $\bar{\theta}>0$ such that $g=f^{-1}$ exists on $(0, \bar{\theta})$ and has the property that $g^{(k)}\left(0^{+}\right)$exists for all $k \geq 1$. Moreover, if $f$ is continuous at zero, $f$ is in $\mathcal{C}^{\infty}$ on $\left[0, \mu^{*}\right)$ and $g$ is in $\mathcal{C}^{\infty}$ on $[0, \bar{\theta})$.

Proof. Let $f$ be as above. The general inverse function theorem [24] establishes that $g=f^{-1}$ exists and is in $\mathcal{C}^{\infty}$ on $(0, \bar{\theta})$, for some $\bar{\theta}>0$. For all $\theta \in(0, \bar{\theta})$,

$$
g^{\prime}(\theta)=\frac{1}{f^{\prime}(g(\theta))}
$$

and since $f^{\prime}(0+)>0$,

$$
g^{\prime}(0+)=\frac{1}{f^{\prime}(g(0+))},
$$

which completes the first statement when $k=1$.

Applying (3.14) on (3.15) yields an expression for $g^{(k)}(\theta)$ in terms of $g^{\prime}(\theta), g^{\prime \prime}(\theta), g^{\prime \prime \prime}(\theta), \ldots$, $g^{(k-1)}(\theta)$, and $f^{\prime}(g(\theta)), f^{\prime \prime}(g(\theta)), f^{\prime \prime \prime}(g(\theta)), \ldots, f^{(k)}(g(\theta))$. So, a simple induction argument shows that $g^{(k)}(0+)$ exists for all $k$. 
Now, suppose that $f$ is continuous at zero, and let $t(\mu)$ be from the mean value theorem. Then

$$
\frac{f(\mu)-f(0)}{\mu}=f^{\prime}(t(\mu)) \text { for all } \mu \in\left(0, \mu^{*}\right) .
$$

Then using the assumption that $f^{\prime}(0+)$ exists implies

$$
\lim _{\mu \downarrow 0}\left|\frac{f(\mu)-f(0)}{\mu}-f^{\prime}(\mu)\right|=\lim _{\mu \downarrow 0}\left|f^{\prime}(t(\mu))-f^{\prime}(\mu)\right|=0 .
$$

So, $f_{+}^{\prime}(0)=f^{\prime}(0+)$ and we have that $f^{\prime}$ is continuous at zero. Repeated applications of the mean value theorem give that $f$ is in $\mathcal{C}^{\infty}$ on $\left[0, \mu^{*}\right)$. Since $f$ being continuous at zero implies $g$ is continuous at zero, a similar argument shows $g$ is in $\mathcal{C}^{\infty}$ on $[0, \bar{\theta})$.

Q.E.D.

We now prove that if $\delta b \in \mathcal{H}_{b} \backslash \mathcal{H}_{b}^{1}$, the primal central optimal solution is not only continuous, but is infinitely, continuously, one-sided differentiable with respect to the perturbation parameter $\theta_{b}$.

Theorem 3.3 $D_{\theta_{b}}^{k} x^{*}\left(0^{+}, 0\right)$ exists for $k=0,1,2, \ldots$, and $D_{\theta_{b}}^{k} x^{*}\left(0^{+}, 0\right)=D_{\theta_{b}^{+}}^{k} x^{*}(0,0)$.

Proof. The case when $\varnothing b \in \mathcal{H}_{b}^{1}$ is already addressed in Theorem 3.2 , so assume that $\varnothing b \in \mathcal{H}_{b} \backslash \mathcal{H}_{b}^{1}$. Let $\theta_{b}^{*}>0$ be such that $\left(B\left(\theta_{b}, 0\right), N\left(\theta_{b}, 0\right)\right)$ is invariant for $\theta_{b} \in\left(0, \theta_{b}^{*}\right)$, and denote this common partition by $\left(B^{\prime} \mid N^{\prime}\right)$. From Lemma 3.1, $B(0,0) \subset B^{\prime}$ and $N^{\prime} \subset N(0,0)$. Therefore, if $i \in N^{\prime}$, $x_{i}^{*}\left(\theta_{b}, 0\right)=0$ for all $\theta_{b} \in\left[0, \theta_{b}^{*}\right)$. Hence, $D_{\theta_{b}}^{k} x_{N^{\prime}}^{*}\left(0^{+}, 0\right)=D_{\theta_{b}^{+}}^{k} x_{N^{\prime}}^{*}(0,0)=0$ for all $k$.

Let $i \in B^{\prime}$, and consider the following linear program

$$
\min \left\{\theta_{b}: A_{B(0,0)} z_{B(0,0)}+A_{\mathcal{I}} z_{\mathcal{I}}-\delta b \theta_{b}=b, z_{B(0,0)} \geq 0, z_{\mathcal{I}} \geq 0, \theta_{b} \geq 0\right\}
$$

where $\mathcal{I}=B^{\prime} \backslash B(0,0)$. Furthermore, let $\left\{\left(z_{B(0,0)}(\mu), z_{\mathcal{I}}(\mu), \theta_{b}(\mu)\right): \mu \geq 0\right\}$ be the central path for this linear program. Then,

$$
z_{B(0,0)}(\mu)=x_{B(0,0)}^{*}\left(\theta_{b}(\mu), 0\right) \text { and } z_{\mathcal{I}}(\mu)=x_{\mathcal{I}}^{*}\left(\theta_{b}(\mu), 0\right) .
$$

Since the optimal value of the linear program is zero, $\theta_{b}(\mu) \rightarrow 0$, as $\mu \downarrow 0$, and from Lemma 3.2, $\theta_{b}^{\prime}(0+)>0$. So, there exists some interval, say $[0, \hat{\mu})$, where $\theta_{b}(\mu)$ is invertible. Denote the inverse by $\mu\left(\theta_{b}\right)$ and let the corresponding interval be $\left[0, \hat{\theta_{b}}\right)$, where we assume that $\hat{\theta}_{b} \leq \theta_{b}^{*}$. Then for all $\theta_{b} \in\left(0, \hat{\theta}_{b}\right)$ we have

$$
z_{B(0,0)}\left(\mu\left(\theta_{b}\right)\right)=x_{B(0,0)}^{*}\left(\theta_{b}, 0\right), z_{\mathcal{I}}\left(\mu\left(\theta_{b}\right)\right)=x_{\mathcal{I}}^{*}\left(\theta_{b}, 0\right)
$$

Applying (3.14) we have the following for $i \in B^{\prime}$,

$$
D_{\theta_{b}}^{k} x_{i}\left(\theta_{b}\right)=\sum_{m=1}^{k} \sum \frac{k !}{j_{1} ! j_{2} ! \ldots j_{k} !} \cdot\left[\left(\frac{\mu^{\prime}\left(\theta_{b}\right)}{1 !}\right)^{j_{1}}\left(\frac{\mu^{\prime \prime}\left(\theta_{b}\right)}{2 !}\right)^{j_{2}} \ldots\left(\frac{\mu^{(k)}\left(\theta_{b}\right)}{k !}\right)^{j_{k}}\right] \cdot z_{i}^{(m)}\left(\mu\left(\theta_{b}\right)\right) .
$$


Lemmas 3.2 and 3.3 imply that the right-hand side converges as $\theta_{b} \downarrow 0$. So, $D_{\theta_{b}}^{k} x^{*}\left(0^{+}, 0\right)$ exists. Now, $z$ is continuous continuous at zero because of the convergence properties of the analytic central path, and $\mu$ is continuous at zero because it is the inverse of $\theta_{b}$, which is in $\mathcal{C}^{\infty}$ and increasing on $[0, \hat{\mu})$. Hence, $x^{*}\left(\theta_{b}, 0\right)$ is continuous at $\theta_{b}=0$, and the mean value theorem is used as in the proof of Lemma 3.3 to conclude the result.

Q.E.D.

Theorems 3.3 and 3.2 have shown the existence of the (one-sided)derivatives of $x^{*}\left(\theta_{b}, 0\right)$. We now conclude this section by showing that these derivatives are uniformly bounded. Let $\mu>0$ and consider the system (2.2), -i.e. the definition of the parametric central path. Using the full rank matrix $\bar{A}$, we have that differentiating with respect to $\theta_{b}$ yields

$$
\begin{aligned}
\bar{A}\left(D_{\theta_{b}}^{1} x\left(\theta_{b}, 0, \mu\right)\right) & =\overline{\delta b} \\
\bar{A}^{T}\left(D_{\theta_{b}}^{1} \bar{y}\left(\theta_{b}, 0, \mu\right)\right)+D_{\theta_{b}}^{1} s\left(\theta_{b}, 0, \mu\right) & =0 \\
S\left(D_{\theta_{b}}^{1} x\left(\theta_{b}, 0, \mu\right)\right)+X\left(\theta_{b}, 0, \mu\right)\left(D_{\theta_{b}}^{1} s\left(\theta_{b}, 0, \mu\right)\right) & =0 .
\end{aligned}
$$

This system implies

$$
D_{\theta_{b}}^{1} x\left(\theta_{b}, 0, \mu\right)=X^{2}\left(\theta_{b}, 0, \mu\right) \bar{A}^{T}\left(\bar{A} X^{2}\left(\theta_{b}, 0, \mu\right) \bar{A}^{T}\right)^{-1} \bar{\delta} .
$$

The equality in (3.16) is convenient because Dikin [6] showed that for a given full row-rank matrix, $M$, the following condition number, $\chi_{M}$, is finite (also see Stewart [31], Todd [32], and for the rank deficient case, Holder and Caron [17]):

$$
\chi_{M} \equiv \sup \left\{\left\|\left(M D M^{T}\right)^{-1} M D\right\|: D \text { is positive diagonal }\right\} .
$$

This condition number, together with (3.11) and (3.16), provides a uniform bound on the first order derivatives.

Theorem 3.4 It holds for any positive $\mu$ that

$$
\left\|D_{\theta_{b}}^{1} x\left(\theta_{b}, 0, \mu\right)\right\| \leq \chi_{\bar{A}}\|\bar{\delta}\|
$$

Furthermore, if $\delta b \in \mathcal{H}_{b}^{1}$,

$$
\left\|D_{\theta_{b}}^{1} x^{*}(0,0)\right\| \leq \chi_{\bar{A}}\|\bar{\delta}\|
$$

Otherwise $\delta b \in \mathcal{H}_{b} \backslash \mathcal{H}_{b}^{1}$, and

$$
\left\|D_{\theta_{b}^{+}}^{1} x^{*}(0,0)\right\| \leq \chi_{\bar{A}}\|\bar{\delta} b\|
$$

Proof. Both (3.17) and (3.18) follow directly equations 3.11 and 3.16, and from the definition of $\chi_{\bar{A}}$. The bound in (3.19) follows since Theorem 3.3 implies $D_{\theta_{b}^{+}}^{1} x^{*}(0,0)=\lim _{\theta_{b} \downarrow 0} D_{\theta_{b}}^{1} x^{*}\left(\theta_{b}, 0\right)$, and form (3.18) the right side of this equality is bounded by $\chi_{\bar{A}}\|\bar{\delta}\|$.

Q.E.D. 
We remark that the bounds in Theorem 3.4 depend only on the $A$-matrix and $\|\delta\|$; in particular, they are independent of $\mu, \theta_{b}$, and $b$. Furthermore, $\|\delta b\|$ can obviously be normalized to one.

The following corollary lists several order relations that follow from Theorems 3.3 and 3.4. This corollary highlights the fact that the entire primal central path behaves "smoothly" with respect to right-hand side parameterization. An extension of the big-O nation is used, viz. $u(t)=\Theta(w(t))$ if $u(t)$ and $w(t)$ are positive sequences, and the ratios $u(t) / w(t)$ and $w(t) / u(t)$ are both bounded.

Corollary 3.2 For all $\mu>0$ and $\theta_{b}$ sufficiently small,

$$
\left\|x\left(\theta_{b}, 0, \mu\right)-x(0,0, \mu)\right\|=O\left(\theta_{b}\right)
$$

and also, since the constant in the big- $O$ is independent of $\mu$,

$$
\left\|x^{*}\left(\theta_{b}, 0\right)-x^{*}(0,0)\right\|=O\left(\theta_{b}\right) .
$$

Furthermore, for sufficiently small $\theta_{b}$, and $\mathcal{I}$ and $N^{\prime}$ as in the proof Theorem 3.3, we have

$$
\begin{aligned}
x_{\mathcal{I}}^{*}\left(\theta_{b}, 0\right) & =\Theta\left(\theta_{b}\right), \\
x_{N^{\prime}}\left(\theta_{b}, 0, \mu\right) & =\Theta(\mu) .
\end{aligned}
$$

Proof. Equations (3.20) and (3.21) follow immediately from the uniform bound presented in (3.17). Equation (3.22) follows from the fact that $x_{\mathcal{I}}^{*}(0,0)=0$ and Lemma 3.2 shows $D_{\theta_{b}^{+}}^{1} x_{\mathcal{I}}(0,0)>0$. To show equation (3.23) we first recognize that from Lemma 3.2, $D_{\mu}^{1} x_{N^{\prime}}\left(\theta_{b}, 0,0^{+}\right)=\left(S_{N^{\prime}}^{*}\left(\theta_{b}, 0\right)\right)^{-1} e_{N^{\prime}}$. Theorem 3.1 implies that $s_{N^{\prime}}^{*}\left(\theta_{b}, 0\right)$ is constant over some suitably small neighborhood, say $\left(0, \theta_{b}^{*}\right)$. Furthermore, the definition of $N^{\prime}$ implies $s_{N^{\prime}}\left(\theta_{b}, 0\right)$ and $s_{N^{\prime}}(0,0)$ are both positive. So over $\left[0, \theta_{b}^{*}\right)$, $D_{\mu}^{1} x_{N^{\prime}}\left(\theta_{b}, 0,0^{+}\right)$is uniformly bounded, which establishes (3.23).

Q.E.D.

The bounds indicated in the last corollary are improvements over those in Theorem 4.1 of [25] by Nunez and Freund, where the upper bound on $\left\|x\left(\theta_{b}, \theta_{c}, \mu\right)-x(0,0, \mu)\right\|$ not only depends on $\mu$, but increases to infinity as $\mu \downarrow 0$. The reason why our results are stronger is that we allow only right-hand side changes, whereas the work of Nunez and Freund allows simultaneous changes in $A$, $b$, and $c$.

\section{Convergence and Order Relations Under Parameterization}

Each of the three results of this section establishes conditions under which the parameterized central path converges. The first of these results establishes that the parameterized central path converges to the unperturbed central optimal solution, provided that only right-hand side perturbation is 
allowed. This result follows directly from a result in [5] and is quite useful because cost coefficient perturbations may also be modeled as right-hand side changes. Modeling cost coefficient changes as right-hand side changes allows us to include simultaneous perturbations in the second and third results. Specifically, the second result shows that if the cost parameter and barrier parameter are scalars of each other, the parameterized central path converges to an element of a central path defined on the unperturbed optimal set. The third result shows that the parameterized central path converges to the unperturbed central optimal solution if the barrier parameter converges to zero slower than the cost coefficient parameter. The following example illustrates all three convergence results and demonstrates that convergence is not always assured.

Example: Consider the linear program $\min \left\{\theta_{c}\left(10 x_{1}\right)+\theta_{c} x_{2}+x_{3}: 0 \leq x_{1}, x_{2}, x_{3} \leq 1+\theta_{b}\right\}$, for which $b=\delta b=(1,1,1)^{T}, c=(0,0,1)^{T}$, and $\delta c=(10,1,0)^{T}$. The parameterized central path is defined by

$$
\begin{aligned}
& x_{1}\left(\theta_{b}, \theta_{c}, \mu\right)= \begin{cases}\frac{1}{20 \theta_{c}}\left(10\left(1+\theta_{b}\right) \theta_{c}+2 \mu-\sqrt{100\left(1+\theta_{b}\right)^{2} \theta_{c}^{2}+4 \mu^{2}}\right) & , \theta_{c} \neq 0 \\
\frac{1+\theta_{b}}{2} & , \theta_{c}=0\end{cases} \\
& x_{2}\left(\theta_{b}, \theta_{c}, \mu\right)= \begin{cases}\frac{1}{2 \theta_{c}}\left(\left(1+\theta_{b}\right) \theta_{c}+2 \mu-\sqrt{\left(1+\theta_{b}\right)^{2} \theta_{c}^{2}+4 \mu^{2}}\right) & , \theta_{c} \neq 0 \\
\frac{1+\theta_{b}}{2} & , \theta_{c}=0\end{cases} \\
& x_{3}\left(\theta_{b}, \theta_{c}, \mu\right)=\frac{1}{2}\left(\left(1+\theta_{b}\right)+2 \mu-\sqrt{\left(1+\theta_{b}\right)^{2}+4 \mu^{2}}\right)
\end{aligned}
$$

To illustrate the next three results, we consider the situations of $\left(\theta_{b}, \theta_{c}\right)=\left(\theta_{b}, 0\right),\left(\theta_{b}, \nu \theta_{c}\right)=\left(\theta_{b}, \mu\right)$, and $\left(\theta_{b}, \theta_{c}\right)=\left(\theta_{b}, \mu^{2}\right)$.

Setting $\theta_{c}$ to 0 , we see that the limit as $\left(\theta_{b}, \mu\right) \downarrow 0$ of the parameterized central path is

$$
\left(x_{1}^{*}(0,0), x_{2}^{*}(0,0), x_{3}^{*}(0,0)\right)=(1 / 2,1 / 2,0) .
$$

This is because the entire central path, including the analytic center solution, shifts "smoothly" as the right-hand side is perturbed. Figure 3 depicts this situation, and a formal convergence result is stated in Theorem 4.1

The second, and most difficult situation to explain, makes the substitutions $\mu=\nu \theta_{c}$. Under this substitution we have that the limits as $\left(\theta_{b}, \theta_{c}\right) \downarrow 0$ are

$$
\begin{aligned}
& x_{1}=\frac{1}{20}\left(10+2 \nu-\sqrt{100+4 \nu^{2}}\right), \\
& x_{2}=\frac{1}{2}\left(1+2 \nu-\sqrt{1+4 \nu^{2}}\right), \text { and } \\
& x_{3}=0 .
\end{aligned}
$$

It so happens that these limits are the $\nu$ element of the central path associated with the linear program $\max \left\{10 x_{1}+x_{2}: 0 \leq x_{1}, x_{2} \leq 1, x_{3}=0\right\}$, or equivalently $\max \left\{10 x_{1}+x_{2}:\left(x_{1}, x_{2}, x_{3}\right) \in \mathcal{P}_{(0,0)}^{*}\right\}$. So, 
not only does the linear relationship between $\mu$ and $\theta_{c}$ allows the convergence of the parameterized central path, but the limit is an element of the central path contained in the optimal set and defined by the cost coefficient direction of change. This special type of convergence is illustrated by Figure 4 Making the substitution $\theta_{c}=\mu^{2}$ and allowing $\left(\theta_{b}, \mu\right) \downarrow 0$, we find that the limit is the analytic center solution of the unperturbed problem -i.e. $\left(x_{1}^{*}(0,0), x_{2}^{*}(0,0), x_{3}^{*}(0,0)\right)=(1 / 2,1 / 2,0)$. As shown in Theorem 4.3, this follows because $\theta_{c}$ converges to zero faster than $\mu$. An example is found in Figure 5.

Notice that a mixture of the last two cases may easily produce a sequence that does not allow convergence. For example, let $\mu^{k}=1 / k$, and $\theta_{c}^{k}$ be $\left(\mu^{k}\right)^{2}$ or $(1 / 2) \mu^{k}$, depending on whether $k$ is even or odd, respectively. Then, the subsequence of even indices converges to $(1 / 2,1 / 2,0)$ and the subsequence of odd indices converges to $\left(\frac{14-\sqrt{116}}{20}, \frac{5-\sqrt{17}}{2}, 0\right)$. The sporadic behavior of this path is shown in Figure 6

Before continuing, we explain the differences between the results of this section and those found in [4] by Bonnans and Potra, in [21] by Mizuno, Todd, and Ye, and in [23] by Monteiro and Tsuchiya. Bonnans and Potra consider a single shifted analytic center in a specific algorithmic framework (see Theorem 4.13 in [4]). Our results are algorithm independent and deal not only with a single shifted analytic center, but rather with a central path defined on the unperturbed optimal set. Furthermore, our results allow simultaneous parameterization of both $b$ and $c$, which is not allowed in the horizontal linear complementary framework they address. Mizuno, Todd, and Ye provide conditions under which the cluster points of the parameterized central path are contained in either the boundary or the relative interior of the unperturbed optimal set (see Section 5 of [21]), whereas our results establish the existence of a unique limit. Mizuno and Tsuchiya show that $x(0, \mu, \mu)$ converges, as $\mu \downarrow 0$ (see Section 4 of [23]). This is a special case of Theorem 4.2, where we establish the convergence of $x\left(\theta_{b}, \theta_{c}, \nu \theta_{c}\right)$, for $\nu>0$ and $\left(\theta_{b}, \theta_{c}\right) \downarrow 0$.

Our development begins with two lemmas, the first of which shows that the analytic center is a continuous function of the right-hand side data. This result follows directly from Theorem 3.1 in [5], where simultaneous changes in the $A$ matrix are also allowed. As already mentioned, this result is handy because cost coefficient changes may be modeled as right-hand side changes, and hence, subsequent results involving convergence under cost coefficient perturbations may include simultaneous data perturbations without extra effort.

Lemma 4.1 (Caron, Greenberg, and Holder [5]) Let $(D \mid F)$ be a partition of $\{1,2, \ldots, n\}$. Then, the argument maximum of

$$
\left\{\sum_{i \in D} \ln \left(x_{i}\right): A x=b, x_{D}>0, x_{F}=0\right\}
$$

is continuous with respect to $b$. 


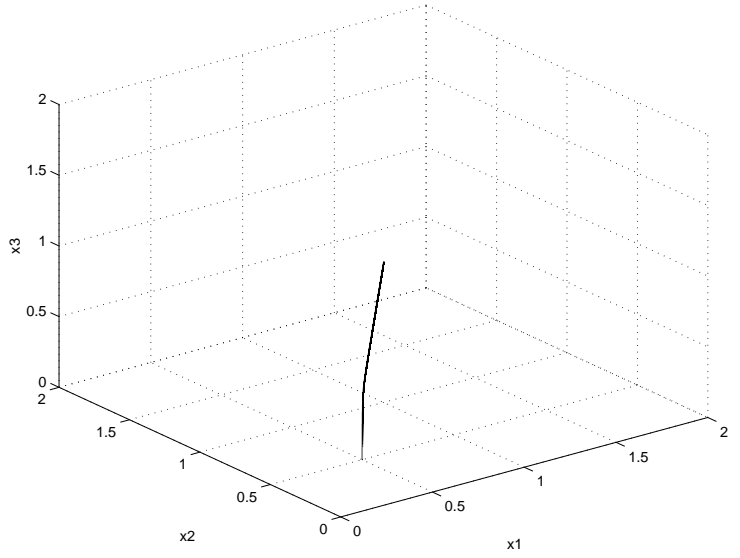

Figure 3: The parameterized central path for $\theta_{c}=0$ and $\theta_{b}$ moving from 1 to 0

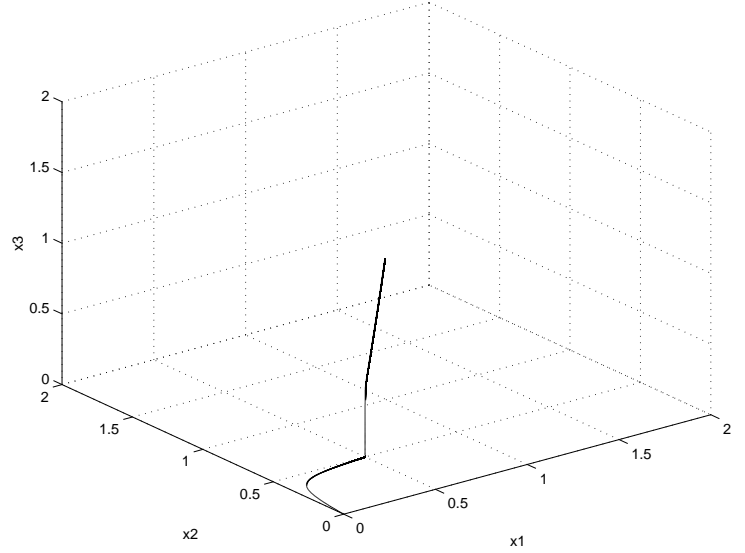

Figure 5: The parameterized central path for $\theta_{b}$ moving from 1 to 0 and $\theta_{c}$ being set to the square of the barrier parameter. The central path in the plane with $x_{3}=0$ is associated with minimizing $\delta c_{B(0,0)} x_{B(0,0)}$ over the unit square.

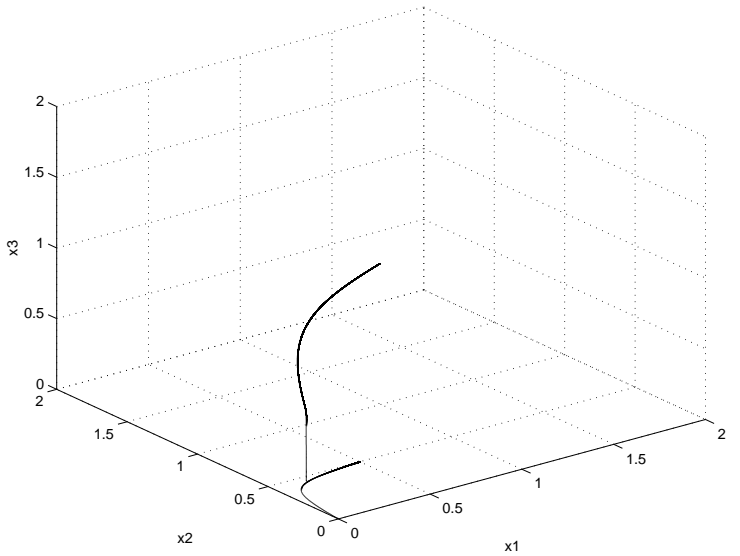

Figure 4: The parameterized central path for $\theta_{b}$ moving from 1 to 0 and $\theta_{c}$ being set to half the barrier parameter. The central path in the plane with $x_{3}=0$ corresponds to minimizing $\delta c_{B(0,0)} x_{B(0,0)}$ over the unit square.

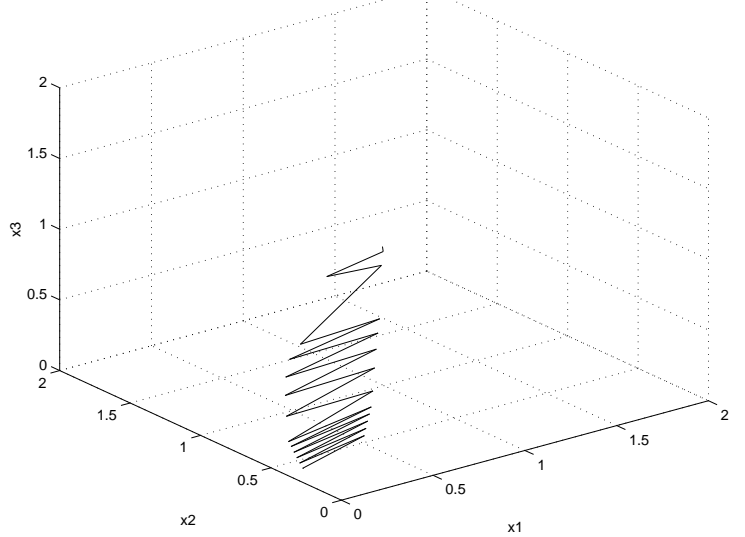

Figure 6: The parameterized central path for $\theta_{b}$ moving from 1 to 0 and $\theta_{c}$ alternating between half the barrier parameter and the square of the barrier parameter.

The second lemma extends Lemma I.6 in [27] to include the situation of data perturbations. This result establishes that the union of the level sets of the primal and dual parameterized central path 
is bounded. These level sets are defined for each $M \geq 0$ as follows,

$$
\mathcal{L}_{M}^{k} \equiv\left\{(x, s):(x,(y, s)) \in \mathcal{P}_{\theta_{b}^{k}} \times \mathcal{D}_{\theta_{c}^{k}}, x^{T} s \leq M\right\}
$$

where $\left(\theta_{b}^{k}, \theta_{c}^{k}\right)$ is any non-negative sequence of parameters.

Lemma 4.2 Let $\left(\theta_{b}^{k}, \theta_{c}^{k}\right) \downarrow 0$, as $k \uparrow \infty$. Then, for all $M \geq 0$,

$$
\bigcup_{k} \mathcal{L}_{M}^{k}
$$

is bounded.

Proof. For any $k$, let $\left(x^{k},\left(y^{k}, s^{k}\right)\right) \in \mathcal{P}_{\theta_{b}^{k}}^{o} \times \mathcal{D}_{\theta_{c}^{k}}^{o}$. Choose $(x, s) \in \mathcal{L}_{M}^{k}$. Then $s^{k}-s \in \operatorname{row}(A)$ and $x^{k}-x \in \operatorname{null}(A)$. So

$$
0=\left(x-x^{k}\right)^{T}\left(s-s^{k}\right)=x^{T} s-x^{T} s^{k}-\left(x^{k}\right)^{T} s+\left(x^{k}\right)^{T} s^{k} .
$$

Non-negativity yields

$$
x_{i} s_{i}^{k} \leq x^{T} s^{k} \leq x^{T} s+\left(x^{k}\right)^{T} s^{k} \leq M+\left(x^{k}\right)^{T} s^{k} .
$$

Hence,

$$
x_{i} \leq \frac{M+\left(x^{k}\right)^{T} s^{k}}{s_{i}^{k}} .
$$

Similarly,

$$
s_{i} \leq \frac{M+\left(x^{k}\right)^{T} s^{k}}{x_{i}^{k}} .
$$

So, for any fixed $k$ and $M \geq 0, \mathcal{L}_{M}^{k}$ is bounded.

Let $\bar{\mu}>0$ and set,

$$
s^{k}=s(0,0, \bar{\mu})+\theta_{c}^{k} \delta c \text { and } x^{k}=x(0,0, \bar{\mu})+\theta_{b}^{k} A^{+} \delta b,
$$

where $A^{+}$is the Moore-Penrose pseudo inverse of $A$. Since $x(0,0, \bar{\mu})$ and $s(0,0, \bar{\mu})$ are positive, there exists a natural number $K^{1}$ such that for all $k \geq K^{1}, s^{k}>0$ and $x^{k}>0$. Furthermore, $A^{T} y(0,0, \bar{\mu})+$ $s^{k}=c+\theta_{c}^{k} \delta c$ and because $\delta b \in \mathcal{H}_{b} \Rightarrow \delta b \in \operatorname{coll}(A), A x^{k}=b+\theta_{b}^{k} \delta b$. So, $\left(x^{k},\left(y(0,0, \bar{\mu}), s^{k}\right)\right) \in$ $\mathcal{P}_{\theta_{b}^{k}}^{o} \times \mathcal{D}_{\theta_{c}^{k}}^{o}$, for all $k \geq K^{1}$. Since $\left(\theta_{b}^{k}, \theta_{c}^{k}\right) \downarrow(0,0)$ as $k \uparrow \infty$, we have that for $k \geq K^{1},\left(x^{k}, s^{k}\right) \geq \alpha>0$, for some $\alpha$. Hence, both

$$
\frac{M+\left(x^{k}\right)^{T} s^{k}}{s_{i}^{k}} \text { and } \frac{M+\left(x^{k}\right)^{T} s^{k}}{x_{i}^{k}}
$$

are bounded for all $k \geq K^{1}$. This implies that

$$
\bigcup_{k \geq K^{1}} \mathcal{L}_{M}^{k}
$$


is bounded. Since

$$
\bigcup_{1 \leq k<K^{1}} \mathcal{L}_{M}^{k}
$$

is a finite union of bounded sets, the result follows.

Although the prior example demonstrates that convergence of the parameterized central path is not guaranteed, Lemma 4.2 assures us that if $\left\{\left(\theta_{c}^{k}, \theta_{b}^{k}, \mu^{k}\right)\right\}$ decreases to zero, both $x\left(\theta_{c}^{k}, \theta_{b}^{k}, \mu^{k}\right)$ and $s\left(\theta_{c}^{k}, \theta_{b}^{k}, \mu^{k}\right)$ have at least one cluster point.

We conclude this section with three convergence results that explain situations guaranteeing the convergence of the parameterized central path. The first of these results shows that the parameterized central path converges to the analytic center solution of the unperturbed linear program, provided there is no change in the cost vector.

Theorem 4.1 It holds that

$$
\lim _{\left(\theta_{b}, \mu\right) \downarrow 0} x\left(\theta_{b}, 0, \mu\right)=x^{*}(0,0)
$$

Proof. The third equality in (2.2) implies $x^{T}\left(\theta_{b}, 0, \mu\right) s\left(\theta_{b}, 0, \mu\right)=n \mu$, and hence Lemma 4.2 implies that $\left\{\left(x\left(\theta_{b}^{k}, 0, \mu^{k}\right), s\left(\theta_{b}^{k}, 0, \mu^{k}\right):\left(\theta_{b}^{k}, \mu^{k}\right) \downarrow 0\right\}\right.$ is bounded. Without loss in generality, let $\left(\theta_{b}^{k}, \mu^{k}\right)$ be such that $\left(x\left(\theta_{b}^{k}, 0, \mu^{k}\right), s\left(\theta_{b}^{k}, 0, \mu^{k}\right)\right) \rightarrow(\hat{x}, \hat{s})$. Since $\hat{x}^{T} \hat{s}=0$, we have that $\hat{x} \in \mathcal{P}^{*}$, which implies $\hat{x}_{N(0,0)}=0$.

To complete the proof we must show that $\hat{x}_{B(0,0)}=x_{B(0,0)}^{*}(0,0)$. First, note that $x_{B(0,0)}\left(\theta_{b}^{k}, 0, \mu^{k}\right)$ is the analytic center of

$$
\left\{x_{B(0,0)}: A_{B(0,0)} x_{B(0,0)}=b+\theta_{b} \delta b-A_{N(0,0)} x_{N(0,0)}\left(\theta_{b}^{k}, 0, \mu^{k}\right), x_{B(0,0)} \geq 0\right\} .
$$

From Lemma 4.1, this analytic center is a continuous function of the right-hand side, $b+\theta_{b} \delta b-$ $A_{N(0,0)} x_{N(0,0)}\left(\theta_{b}^{k}, 0, \mu^{k}\right)$. Now, since $b+\theta_{b} \delta b-A_{N(0,0)} x_{N(0,0)}\left(\theta_{b}^{k}, 0, \mu^{k}\right) \rightarrow b$, we have that

$$
x_{B(0,0)}\left(\theta_{b}^{k}, 0, \mu^{k}\right) \rightarrow \hat{x}_{B(0,0)}=x_{B(0,0)}^{*}(0,0) .
$$

Q.E.D.

While Theorem 4.24 shows that the parameterized central path is continuous at $(0,0,0)$, with respect to both the barrier parameter and the right-hand side parameter, we have already seen in the previous example that a similar continuity result is not possible with respect to the cost coefficient parameter. However, the next result shows that convergence is assured as long as the barrier parameter and the cost coefficient parameter are linearly related. Furthermore, the limit under this special parameterization is an element of the central path contained in the unperturbed optimal set and defined by the cost coefficient direction of change. The elements of this central 
path are denoted by $z(b, \nu)$ and are the unique solutions to

$$
\max \left\{\delta c_{B} z-\nu \sum_{i \in B} \ln \left(z_{i}\right): A_{B} z=b, z>0\right\} .
$$

This central path inherits the analytic properties of any other central path. Specifically, we require the use that for any $\nu>0$,

$$
\lim _{b \rightarrow \bar{b}} z(b, \nu)=z(\bar{b}, \nu)
$$

Theorem 4.2 For any given parameter $\nu>0$, it holds that

$$
\lim _{\left(\theta_{b}, \theta_{c}\right) \rightarrow 0}\left(x_{B}\left(\theta_{b}, \theta_{c}, \theta_{c} \nu\right), x_{N}\left(\theta_{b}, \theta_{c}, \theta_{c} \nu\right)\right)=(z(b, \nu), 0) .
$$

Proof. From Lemma 4.2,

$$
\left\{\left(x\left(\theta_{b}^{k}, \theta_{c}^{k}, \theta_{c}^{k} \nu\right), s\left(\theta_{b}^{k}, \theta_{c}^{k}, \theta_{c}^{k} \nu\right)\right):\left(\theta_{b}^{k} \theta_{c}^{k}\right) \downarrow 0\right\}
$$

is bounded. Since

$$
x^{T}\left(\theta_{b}^{k}, \theta_{c}^{k}, \theta_{c}^{k} \nu\right) s\left(\theta_{b}^{k}, \theta_{c}^{k}, \theta_{c}^{k} \nu\right)=n \theta_{c}^{k} \nu,
$$

we have that any cluster point of $x\left(\theta_{b}^{k}, \theta_{c}^{k}, \theta_{c}^{k} \nu\right)$, as $\left(\theta_{b}^{k}, \theta_{c}^{k}\right) \downarrow 0$, is in $\mathcal{P}_{(0,0)}^{*}$. Hence,

$$
x_{N(0,0)}\left(\theta_{b}^{k}, \theta_{c}^{k}, \theta_{c}^{k} \nu\right) \rightarrow 0, \text { as }\left(\theta_{b}^{k}, \theta_{c}^{k}\right) \downarrow 0 .
$$

Set

$$
\begin{aligned}
\bar{y}\left(\theta_{b}^{k}, \theta_{c}^{k}, \theta_{c}^{k} \nu\right) & =\frac{1}{\theta_{c}}\left(y\left(\theta_{b}^{k}, \theta_{c}^{k}, \theta_{c}^{k} \nu\right)-y^{*}(0,0)\right) \text { and } \\
\bar{s}\left(\theta_{b}^{k}, \theta_{c}^{k}, \theta_{c}^{k} \nu\right) & =\frac{1}{\theta_{c}}\left(s\left(\theta_{b}^{k}, \theta_{c}^{k}, \theta_{c}^{k} \nu\right)-s^{*}(0,0)\right) .
\end{aligned}
$$

Using that

$$
\begin{aligned}
s_{B(0,0)}^{*}(0,0) & =0, \\
A_{B(0,0)}^{T} y\left(\theta_{b}^{k}, \theta_{c}^{k}, \theta_{c}^{k} \nu\right)+s_{B(0,0)}\left(\theta_{b}^{k}, \theta_{c}^{k}, \theta_{c}^{k} \nu\right) & =c_{B(0,0)}+\theta_{c} \delta c_{B(0,0)}, \text { and } \\
A_{B(0,0)}^{T} y^{*}(0,0) & =c_{B(0,0)},
\end{aligned}
$$

we have that $\left(x\left(\theta_{b}^{k}, \theta_{c}^{k}, \theta_{c}^{k} \nu\right), \bar{y}\left(\theta_{b}^{k}, \theta_{c}^{k}, \theta_{c}^{k} \nu\right), \bar{s}\left(\theta_{b}^{k}, \theta_{c}^{k}, \theta_{c}^{k} \nu\right)\right)$ solves

$$
\begin{aligned}
A_{B(0,0)} x_{B(0,0)} & =b+\theta_{b} \not b-A_{N(0,0)} x_{N(0,0)}\left(\theta_{b}^{k}, \theta_{c}^{k}, \theta_{c}^{k} \nu\right) \\
A_{B(0,0)}^{T} \bar{y}+\bar{s}_{B(0,0)} & =\delta c_{B(0,0)} \\
X_{B(0,0)} \bar{S}_{B(0,0)} e & =\nu e .
\end{aligned}
$$


Since these are the necessary and sufficient Lagrange equations describing

$$
z\left(b+\theta_{b} \varnothing b-A_{N(0,0)} x_{N(0,0)}\left(\theta_{b}^{k}, \theta_{c}^{k}, \theta_{c}^{k} \nu\right), \nu\right),
$$

we have that $x_{B(0,0)}\left(\theta_{b}^{k}, \theta_{c}^{k}, \theta_{c}^{k} \nu\right)=z\left(b+\theta_{b} \delta b-A_{N(0,0)} x_{N(0,0)}\left(\theta_{b}^{k}, \theta_{c}^{k}, \theta_{c}^{k} \nu\right), \nu\right)$. From (4.24), we see that

$$
\lim _{\left(\theta_{b}, \theta_{c}\right) \downarrow 0} x_{B(0,0)}\left(\theta_{b}^{k}, \theta_{c}^{k}, \theta_{c}^{k} \nu\right)=\lim _{\left(\theta_{b}, \theta_{c}\right) \downarrow 0} z\left(b+\theta_{b} \delta b-A_{N(0,0)} x_{N(0,0)}\left(\theta_{b}^{k}, \theta_{c}^{k}, \theta_{c}^{k} \nu\right), \nu\right)=z(b, \nu) .
$$

Q.E.D.

The third and final result of this section shows that if the cost coefficient parameter converges to zero faster than the barrier parameter, the parameterized central path converges to the analytic center solution of the unperturbed linear program.

Theorem 4.3 If $\theta_{c}=o(\mu)$ and $\left(\theta_{b}, \mu\right) \downarrow(0,0)$,

$$
\lim _{\left(\theta_{b}, \theta_{c}, \mu\right) \downarrow 0} x\left(\theta_{b}, \theta_{c}, \mu\right)=x^{*}(0,0) .
$$

\section{Proof.}

Consider a sequence $\left\{\left(\theta_{b}^{k}, \theta_{c}^{k}, \mu^{k}\right): k=1,2, \ldots\right\}$ with

$$
\lim _{k \uparrow \infty} \theta_{b}^{k}=\lim _{k \uparrow \infty} \mu^{k}=\lim _{k \uparrow \infty} \theta_{c}^{k} / \mu^{k}=0 .
$$

Let $F_{k}(x)=\left(c^{T} x / \mu^{k}\right)-\sum_{i=1}^{n} \ln \left(x_{i}\right)$ and notice that $x\left(\theta_{b}, 0, \mu^{k}\right)$ is the unique solution to

$$
\min \left\{F_{k}(x): A x=b+\theta_{b}^{k} \delta b, x>0\right\} .
$$

Assume, for the sake of attaining a contradiction, that

$$
\lim _{k \uparrow \infty}\left(x\left(\theta_{b}, \theta_{c}, \mu\right)-x\left(\theta_{b}, 0, \mu\right)\right) \neq 0 .
$$

The optimality of $x\left(\theta_{b}, \theta_{c}, \mu\right)$ and $x\left(\theta_{b}, 0, \mu\right)$ imply that

$$
F_{k}\left(x\left(\theta_{b}^{k}, 0, \mu^{k}\right)\right) \leq F_{k}\left(x\left(\theta_{b}^{k}, \theta_{c}^{k}, \mu^{k}\right)\right) \leq F_{k}\left(x\left(\theta_{b}^{k}, 0, \mu^{k}\right)\right)-\frac{\theta_{c}^{k}}{\mu^{k}}(\delta c)^{T} x^{k}+\frac{\theta_{c}^{k}}{\mu^{k}}(\delta c)^{T} \hat{x}^{k} .
$$

Observe now that $F_{k}(x)$ is strongly convex and that the eigenvalues of the Hessian matrix of $F_{k}$ are uniformly bounded from zero. Hence, if $\lim _{k \uparrow \infty}\left(\hat{x}^{k}-x^{k}\right) \neq 0$, there exists $\epsilon>0$ such that for each natural number, $N$, there exists $k \geq N$, such that

$$
F_{k}\left(x\left(\theta_{b}^{k}, \theta_{c}^{k}, \mu^{k}\right)\right) \geq F_{k}\left(x\left(\theta_{b}^{k}, 0, \mu^{k}\right)\right)+\epsilon .
$$


However, since $\theta_{c}^{k} / \mu^{k} \rightarrow 0$, this is a contradiction to (4.25). So, we must have

$$
\lim _{k \uparrow \infty}\left(x\left(\theta_{b}^{k}, \theta_{c}^{k}, \mu^{k}\right)-x\left(\theta_{b}^{k}, 0, \mu^{k}\right)\right)=0 .
$$

Using Theorem 4.1, we have

$$
\begin{aligned}
0 & =\lim _{k \uparrow \infty}\left(x\left(\theta_{b}^{k}, \theta_{c}^{k}, \mu^{k}\right)-x\left(\theta_{b}^{k}, 0, \mu^{k}\right)\right) \\
& =\lim _{k \uparrow \infty}\left(x\left(\theta_{b}^{k}, \theta_{c}^{k}, \mu^{k}\right)-x^{*}(0,0)\right)+\lim _{k \uparrow \infty}\left(x^{*}(0,0)-x\left(\theta_{b}^{k}, 0, \mu^{k}\right)\right) \\
& =\lim _{k \uparrow \infty}\left(x\left(\theta_{b}^{k}, \theta_{c}^{k}, \mu^{k}\right)-x^{*}(0,0)\right) .
\end{aligned}
$$

Q.E.D.

\section{Concluding remarks}

In this paper we carried out an investigation of how the parameterized central path and the central optimal solution (analytic center of the optimal set) react to changes in the right-hand side vector, $b$, and the objective vector, $c$. These issues are important in the context of sensitivity analysis and parametric programming.

As was shown, the parameterized central path behaves differently under parameterization of $b$ and $c$. In the former case, we proved that the (primal) central optimal solution has one-sided differentiability with respect to the perturbation parameter. Consequently, the entire central path has a smooth, uniformly bounded shift. Perturbation in the objective vector, however, may cause drastic changes in the central path. In this case we showed that every element of the central path defined on the optimal face is a cluster point of the parametrized central path.

\section{References}

[1] Adler, I. and Monteiro, R.D.C., Limiting behavior of the affine scaling continuous trajectories for linear programming problems, Mathematical Programming 50 (1991) 29-51.

[2] Adler, I. and Monteiro, R.D.C., A geometric view of parametric linear programming, Algorithmica 8 (1992) 161-176.

[3] Berkelaar, A.B., Roos, C., and Terlaky, T., The optimal set and optimal partition approach to linear and quadratic programming. Chapter 6 in In Greenberg, H.J. and Gal, T., eds., Recent advances in sensitivity analysis and parametric programming, Kluwer Publishers, 1997. 
[4] Bonnans, J.F. and Potra, F., On the convergence of the iteration sequence of infeasible path following algorithms for linear complementarity problems, Mathematics of Operations Research 22 (1997) 378-407.

[5] R.J. Caron, H.J. Greenberg, and A.G. Holder, Analytic Centers and Repelling Inequalities, Technical Report CCM\#142, Center for Computational Mathematics, University of Colorado at Denver, 1999.

[6] Dikin, I.I., Iterative solution of problems of linear and quadratic programming, Soviet Mathematics Doklady 8 (1967) 674-675.

[7] Fiacco, A., Introduction to Sensitivity and Stability Analysis in Nonlinear Programming, Academic Press, 1983.

[8] Gradshteyn, I.S. and Ryzhik, I.M., Table of Integrals, Series, and Products, Academic Press, 1979.

[9] Greenberg, H., Computer-Assisted Analysis System for Mathematical Programming Models and Solutions: A User's Guide for ANALYZE, Kluwer, 1993.

[10] Greenberg, H.J., The use of the optimal partition in linear programming solution for postoptimal analysis, Operations research Letters 15 (1994) 179-185.

[11] Greenberg, H., Rim Sensitivity Analysis from an Interior Solution, University of Colorado at Denver, Technical Report, CCM-86, 1996.

[12] Greenberg, H.J. Mathematical Programming Glossary, World Wide Web, http://www . cudenver.edu/ ^hgreenbe/glossary/glossary.html, 1997-99.

[13] Greenberg, H.J. and Gal, T., eds., Recent advances in sensitivity analysis and parametric programming, Kluwer Publishers, 1997.

[14] Greenberg, H.J., Holder, A.G., Roos, C. and Terlaky, T., On the dimension of the set of rim perturbations for optimal partition invariance, SIAM Journal on Optimization 9 (1998) 207 216.

[15] Güler, O., Limiting behavior of weighted central paths in linear programming, Mathematical Programming 65 (1994) 347-363.

[16] Holder, A., Sensitivity Analysis and the Analytic Central Path, Ph.D. Thesis, University of Colorado at Denver, USA (1998).

[17] A.G. Holder, R.J. Caron Uniform Bounds on the Limiting and Marginal Derivatives of the Analytic Center Solution over a Set of Normalized Weights, to appear in OR Letters. 
[18] Jansen, B., Roos, C. and Terlaky, T., An interior point approach to post-optimal and parametric analysis in linear programming. Technical Report 92-21, Faculty of Technical Mathematics and Informatics, TU Delft, The Netherlands, 1992.

[19] L. McLinden, An analogue of Moreau's proximation theorem, with applications to the nonlinear complementary problem, Pacific Journal of Mathematics, 88 (1980) 101-161.

[20] Megiddo, N., Pathways to the optimal set in linear programming, in Progress in Mathematical Programming: Interior-Point and Related Methods, N. Megiddo ed., Springer-Verlag, New York, pp. 131-158, 1989.

[21] Mizuno, S., Todd, M.J. and Ye, Y., A surface of analytic centers and primal-dual infeasibleinterior-point algorithms for linear programming, Mathematics of Operations Research 20 (1995) 135-162.

[22] Monteiro, R.D.C. and Mehrotra, S., A general parametric analysis approach and its implication to sensitivity analysis in interior point methods, Mathematical programming 72 (1996) 65-82.

[23] Monteiro, R.D.C. and Tsuchiya, T., Limiting behavior of the derivatives of certain trajectories associated with a monotone horizontal linear complementarity problem, Mathematics of Operations Research 21 (1996) 793-814.

[24] Munkres, J., Analysis on Manifolds, Addison-Wesley Publishing Company, 1991.

[25] Nunez, M.A. and Freund, R.M., Condition measures and properties of the central trajectory of a linear program, working paper, 1996.

[26] Robinson, S. M., A characterization of stability in linear programming, Operations Research, 25 (1977) 435-447.

[27] Roos, C., Interior point approach to linear programming: theory, algorithms \& parametric analysis, in Topics in Engineering Mathematics, van der Burgh, A. and Simonis, J. eds., Kluwer Academic Publishers, 1992.

[28] Roos, C., Terlaky, T., and Vial, J.-Ph., Theory and Algorithms for Linear Optimization, John Wiley \& Sons, chapter 19, 1997.

[29] Sonnevend, Gy., Applications of the notion of analytic center in approximation (estimation) problems, Journal of Computational and Applied Mathematics, 28 (1989) 349-358.

[30] Sonnevend, Gy., An "Analytic Centre" for polyhedrons and new classes of global algorithms for linear (smooth, convex) programming, in Lecture Notes in Control and Information Sciences, Prékopa, A., Szelezsán, J., and Strazicky, B., eds, vol. 84, 1986. 
[31] Stewart, G.W., On scaled projections and pseudoinverses, Linear Algebraa and Its Applications 112 (1989) 189-193.

[32] Todd, M.J., A Dantzig-Wolfe-like variant of Karmarkar's interior-point linear programming algorithm, Operations Research 38 (1990) 1006-1018.

[33] Witzgall, C., Boggs, P., and Domich, P., On the convergence behavior of trajectories for linear programming, Contemporary Mathematics, 114 (1990) 161-187.

[34] Zhang, S., On the strictly complementary slackness relation in linear programming, Advances in Optimization and Approximation, eds. D.Z. Du and J. Sun, Kluwer, 347-361, 1994. 